\title{
Spectrally Resolved Measurement of Flame Radiation to Determine Soot Temperature and Concentration
}

\author{
D. R. Snelling* \\ National Research Council, Ottawa, Ontario K1A 0R6, Canada \\ K. A. Thomson \\ University of Waterloo, Waterloo, Ontario N2L 3G1, Canada \\ G. J. Smallwood \\ National Research Council, Ottawa, Ontario K1A 0R6, Canada \\ Ö. L. Gülder ${ }^{\S}$ \\ University of Toronto, Toronto, Ontario M3H 5T6, Canada \\ and \\ E. J. Weckman ${ }^{\mathbb{I I}}$ and R. A. Fraser ${ }^{\mathbb{I}}$ \\ University of Waterloo, Waterloo, Ontario N2L 3G1, Canada
}

\begin{abstract}
A multiwavelength flame emission technique is developed for high spatial resolution determination of soot temperature and soot volume fraction in axisymmetric laminar diffusion flames. Horizontal scans of line-integrated spectra are collected over a spectral range of 500-945 nm. Inversion of these data through one-dimensional tomography using a three-point Abel inversion yields radial distributions of the soot radiation from which temperature profiles are extracted. From an absolute calibration of the flame emission and by use of these temperature data, absorption coefficients are calculated, which are directly proportional to the soot volume fractions. The important optical parameters are discussed. It is shown that a uniform sampling cross section through the flame must be maintained and that variations in sampling area produce inconsistencies between measurements and theory, which cannot be interpreted as spatial averaging of the property field. The variations in cross-sectional sampling area have the largest influence on the measurements at the edges of the flame, where the highest resolution is required. Emission attenuation by soot has been shown to have minor influence on the soot temperature and soot volume fraction for the soot loading of the axisymmetric flame tested. An emission correction scheme is outlined, which could be used for more heavily sooting flames. For a refractive index absorption function $E(m)=\operatorname{Im}\left[\left(m^{2}-1\right) /\left(m^{2}+2\right)\right]$ that is independent of wavelength, the soot temperatures and soot volume fractions measured with this technique are in excellent agreement with data obtained by coherent anti-Stokes Raman scattering nitrogen thermometry and two-dimensional soot extinction in the same ethylene coflow diffusion flame. The agreement of the results suggests a limit of the slope of the spectral response of $E(m)$ to be between 0 and $20 \%$ over the spectral range examined.
\end{abstract}

\begin{tabular}{|c|c|c|}
\hline \multicolumn{3}{|r|}{ Nomenclature } \\
\hline$B_{\lambda}$ & $=$ & Planck function, $\mathrm{W} / \mathrm{m}^{3}$ \\
\hline$c$ & $=$ & speed of light in vacuum, $\mathrm{m} / \mathrm{s}$ \\
\hline$d$ & $=$ & diameter of lens aperture, $\mathrm{m}$ \\
\hline$d_{p}$ & $=$ & primary particle diameter, $\mathrm{m}$ \\
\hline$E(m)$ & $=$ & refractive index absorption function for soot \\
\hline$f_{v}$ & $=$ & soot volume fraction \\
\hline$G_{\lambda, i}$ & $=$ & local spectral emission property field, $\mathrm{W} / \mathrm{m}^{4} \cdot \mathrm{sr}$ \\
\hline$h$ & $=$ & Planck's constant, J s \\
\hline$I_{\text {corr }}$ & $=$ & corrected emission measurement, $\mathrm{W} / \mathrm{m}^{2} \cdot \mathrm{sr}$ \\
\hline$I_{\text {meas }}$ & $=$ & emission measurement, $\mathrm{W} / \mathrm{m}^{2} \cdot \mathrm{sr}$ \\
\hline$I_{\text {model }_{\text {ext }}}$ & $=$ & $\begin{array}{l}\text { modeled emission with emission attenuation } \\
\text { accounted for, } \mathrm{W} / \mathrm{m}^{2} \cdot \mathrm{sr}\end{array}$ \\
\hline$I_{\text {model }_{\text {noext }}}$ & $=$ & $\begin{array}{l}\text { modeled emission without emission attenuation } \\
\text { accounted for, } \mathrm{W} / \mathrm{m}^{2} \cdot \mathrm{sr}\end{array}$ \\
\hline$I_{2}$ & $=$ & integrated line-of-sight emission intensity, $\mathrm{W} / \mathrm{m}$ \\
\hline
\end{tabular}

Received 30 August 2001; revision received 22 January 2002; accepted for publication 29 January 2002. Copyright (C) 2002 by the authors. Published by the American Institute of Aeronautics and Astronautics, Inc., with permission. Copies of this paper may be made for personal or internal use, on condition that the copier pay the $\$ 10.00$ per-copy fee to the Copyrigh Clearance Center, Inc., 222 Rosewood Drive, Danvers, MA 01923; include the code 0001-1452/02 \$10.00 in correspondence with the CCC.

* Principal Research Officer, Institute for Chemical Process and Environmental Technology, Combustion Research Laboratory, M-9.

${ }^{\dagger} \mathrm{Ph} . \mathrm{D}$. Candidate, Mechanical Engineering Department.

*Senior Research Officer, Institute for Chemical Process and Environmental Technology, Combustion Research Laboratory, M-9.

${ }^{\S}$ Professor, Institute for Space Studies.

II Associate Professor, Mechanical Engineering Department.

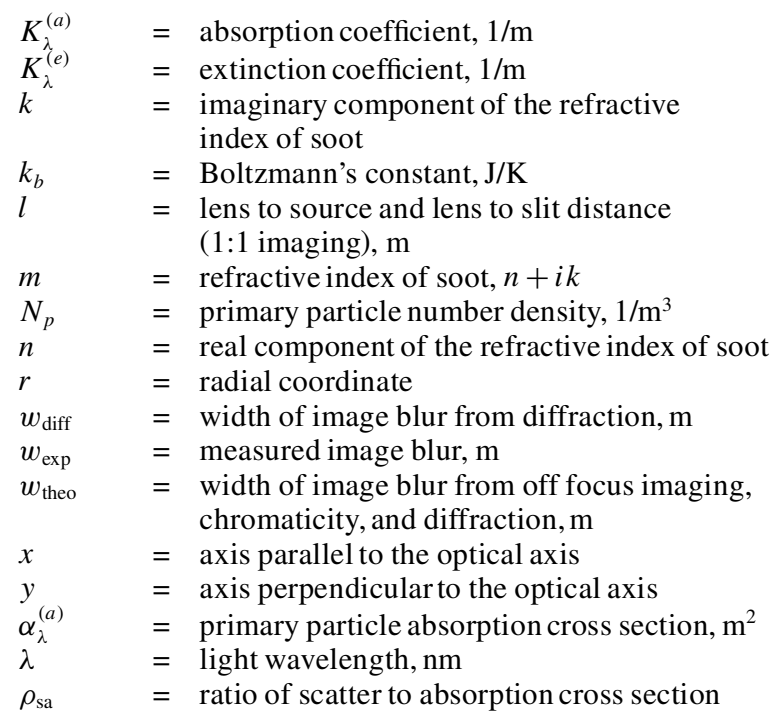

\section{Introduction}

$\mathbf{K}$ NOWLEDGE of soot formation mechanisms and soot optical properties is important in many areas of combustion research. Soot formation directly relates to flame radiation and heat transfer properties, particulate emission from combustion systems, and combustion efficiency. Therefore, nonintrusive optical diagnostics that measure radiant emission from hot soot are a key source of information on the soot fields in many combustion applications. 
Soot temperature and concentration can be obtained from emission/transmission measurements or from multiwavelength emission measurements. Hall and Boncyzk ${ }^{1}$ used single wavelength emission/transmission tomography, with line-of-sight integrated measurements, to reconstruct two-dimensional maps of soot concentration and temperature in a laminar diffusion flame very similar to our own. Two-color analysis of soot radiative emission, the simplest multiwavelength emission diagnostic, has provided data on spatially averaged soot concentration and soot temperature in engines. ${ }^{2}$ The technique has also been applied, using water-cooled fibre-optic probes, to the study of soot properties in flames. ${ }^{3-5}$ DeIuliis et al. ${ }^{6}$ extended the multiwavelength diagnostic, collecting radially resolved multiwavelength flame emission in an axisymmetric flame similar to Hall and Boncyzk. ${ }^{1}$ When the tomographic technique was applied, DeIuliis et al. ${ }^{6}$ did not consider flame absorption of radiation or address the issue of depth-of-field requirements as outlined by Hall and Boncyzk. ${ }^{1}$ The depth of field is the object distance on either side of the focal plane within which the acceptable blur limit is not exceeded.

The present study further develops the soot emission temperature measurement method by analyzing optical conditions dictated by depth of field, greatly increasing the spatial resolution of the technique, demonstrating a correction procedure for flame attenuation of radiation, and evaluating the accuracy of the method through comparisons of the results to coherent anti-Stokes Raman scattering (CARS) temperature data and light-extinction-based soot concentration measurements taken in the same flame at similar high spatial resolution. This comparison of results is used to propose limitations on the possible variation of the refractive index function $E(m)$ with wavelength.

\section{Theory}

The soot emission temperature measurement technique is based on analysis of the radiation emission from soot transmitted along a chord defined by $y=y_{0}$ (Fig. 1) in an axisymmetric flame without external incident radiation. This is described by a modification to the generalized path integral for line-of-sight emission intensity ${ }^{1,7}$ :

$$
\begin{aligned}
I_{\lambda}\left(y_{0}\right) & =\int_{-\infty}^{\infty} N_{P}\left(x, y_{0}\right) \alpha_{\lambda}^{(a)} B_{\lambda}\left[T\left(x, y_{0}\right)\right] \\
& \times \exp \left[-\int_{x}^{\infty} K_{\lambda}^{(e)}\left(x^{\prime}, y_{0}\right) \mathrm{d} x^{\prime}\right] \mathrm{d} x
\end{aligned}
$$

The product of $N_{p} \alpha_{\lambda}^{(a)}$ is equal to $K_{\lambda}^{(a)}$ as follows from RayleighDebye-Gans/polydisperse-fractal aggregate (RDG/PFA) theory of particle light scattering and extinction ${ }^{8-13}$ for primary particles in the Rayleigh range, that is, $\pi d / \lambda<0.3$. The Planck function $B_{\lambda}(T)$ is given by

$$
\begin{aligned}
B_{\lambda}(T) & =\left(2 \pi h c^{2} / \lambda^{5}\right)[\exp (h c / \lambda k T)-1]^{-1} \approx\left(2 \pi h c^{2} / \lambda^{5}\right) \\
\times & \exp [-(h c / \lambda k T)]
\end{aligned}
$$

where the latter approximation is excellent for the wavelengths and temperatures encountered here.

Equation (1) represents a line integration of radiation emission from the soot along the chord $y=y_{0}$. The term before the exponen-

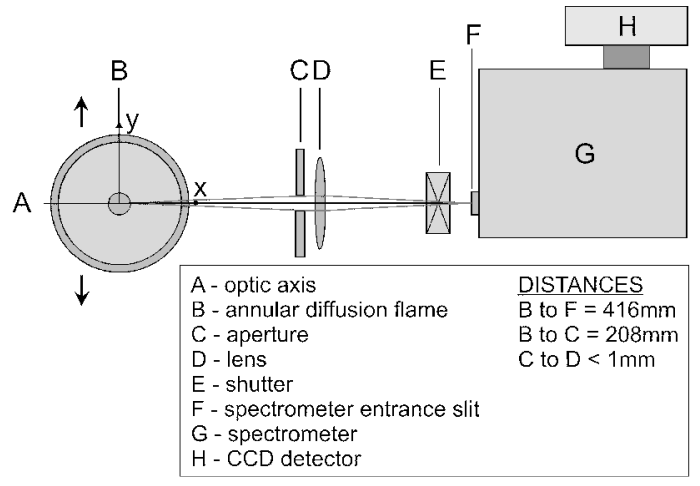

Fig. 1 Schematic of the optical layout. tial, $N_{P}\left(x, y_{0}\right) \alpha_{\lambda}^{(a)} B_{\lambda}\left[T\left(x, y_{0}\right)\right]$, models the local radiation emission rate, and the exponential term,

$$
-\int_{x}^{\infty} K_{\lambda}^{(e)}\left(x^{\prime}, y\right) \mathrm{d} x^{\prime}
$$

models the attenuation experienced by this emitted radiation as it propagates to the detector side of the flame along the chord. The attenuation of the radiation emission is, therefore, dependent on the position of the radiation source in the flame.

As a first approximation to Eq. (1), the attenuation of emitted radiation is ignored, that is,

$$
\int_{x}^{\infty} K_{\lambda}^{(e)}\left(x^{\prime}, y_{0}\right) \mathrm{d} x^{\prime} \approx 0
$$

and, assuming radial symmetry, the tomographic reconstruction recovers the local property field $G_{\lambda, r}$, which is given by

$$
G_{\lambda, r}=N_{p}(r) \alpha_{\lambda}^{(a)} B_{\lambda}[T(r)]
$$

In the Rayleigh limit, the primary particle absorption cross section and its dependence on wavelength (relative to a reference wavelength $\lambda_{c}$ ) are

$$
\alpha_{\lambda}^{(a)}=\frac{\pi^{2} d_{p}^{3} E\left(m_{\lambda}\right)}{\lambda}=\alpha_{\lambda_{c}}^{(a)} \frac{\lambda_{c}}{\lambda} \frac{E\left(m_{\lambda}\right)}{E\left(m_{\lambda_{c}}\right)}
$$

where $E(m)$ is a wavelength-dependentfunction of the complex refractive index $m$ given by $-\operatorname{Im}\left[\left(m^{2}-1\right) /\left(m^{2}+2\right)\right]$. The logarithm of Eq. (3) when combined with Eq. (4) gives

$$
\ln \left[\frac{E\left(m_{\lambda}\right)}{G_{\lambda, r} \cdot \lambda^{6}}\right]=\frac{h c}{\lambda k T(r)}+c_{1}
$$

where all variables from Eqs. (3) and (4) that are constant with $\lambda$ have been grouped as $c_{1}$. An example of the plot of the left-hand side of Eq. (5) vs $1 / \lambda$ is shown in Fig. 2 The slope of this plot, determined by the method of least mean squares, is $h c / k T$, from which $T$ can be extracted. It is this step in the multiwavelength emission diagnostic that distinguishes it from the emission/transmission diagnostic: Through the use of emission measurements from multiple wavelengths, there is no need to estimate the absorption coefficient from transmission (extinction) measurements. The absorption coefficient $N_{p} \alpha_{\lambda}^{(a)}$ is calculated using the calculated temperature and Eq. (3). The soot volume fraction is then estimated using

$$
f_{v}=\frac{N_{p} \alpha_{\lambda}^{(a)} \lambda}{6 \pi E\left(m_{\lambda}\right)}
$$

To calculate the soot volume fraction, the absolute value of $E(m)$ is required, whereas to calculate the soot temperature, the relative variation of $E(m)$ with wavelength is sufficient. The correct value for the $E(m)$ function is much debated. ${ }^{14}$ For the figures, unless stated otherwise, $E(m)$ is taken to be constant, independent of wavelength, ${ }^{15}$ with a magnitude of $0.26(n=1.57$ and $k=0.56)$

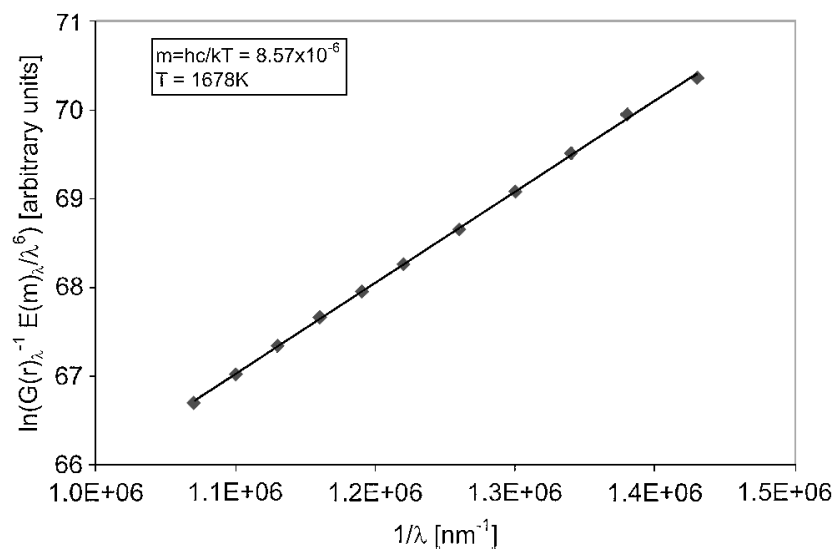

Fig. 2 Example of plot from which temperature is inferred [see Eq. (5)]; height in flame $=30 \mathrm{~mm}$ and radial position $=0 \mathrm{~mm}$ (see Fig. 7). 
(Ref. 16). Note that the present results, and their comparison with light-attenuation-based soot concentrationmeasurements, cannotbe used to infer absolute values of the soot scattering function $E(m)$ For a comparison of the two measurements of soot concentrationto be valid, the same value of $E(m)$ must be used in the analysis of the data. Because the soot concentration from both light attenuation and the present radiation measurement depends linearly on the value of $E(m)$ assumed, their agreement carries no implication as to its value. However, the soot concentration obtained from flame intensities soot emission temperature measurements is very dependent on the temperatures and, hence, on the variation of $E(m)$ with wavelength used to obtain those temperatures. The variation of $E(m)$ with wavelength on the inferred soot temperatures and concentrations is discussed.

\section{Experimental Methodology}

The experimental setup is shown schematically in Fig. 1. Radiation emission from soot in the laminar diffusion flame passes through an adjustable aperture and is focused by a 104-mm-diam achromatic lens onto the entrance slit of a spectrometer. The spectrometer slit is oriented vertically with a height of $0.500 \mathrm{~mm}$ and a width of $0.025 \mathrm{~mm}$. The output from the spectrometer images onto a charge-coupled device (CCD) detector. The optics are set for 1:1 imaging magnification.

The laminar diffusion flame burner is of similar design to that previously reported by the National Research Council of Canada ${ }^{17}$ and others. ${ }^{13,18,19}$ It consists of a 10.9-mm-inner-diameterfuel tube, centred in a 100-mm-diam air nozzle. The air passes through packed beds of glass beads and porous metal disks to smooth the flow and prevent flame instabilities. In the present experiment, the $\mathrm{C}_{2} \mathrm{H}_{4}$ fuel flow rate is set to the smoke point, $194 \mathrm{ml} / \mathrm{min}\left(21^{\circ} \mathrm{C}, 1 \mathrm{~atm}\right)$, and the air coflow is $284 \mathrm{l} / \mathrm{min}$. A flame enclosure made of flexible steel mesh protects the flame from air movements in the room. An appropriate viewing port in the mesh provides optical access. The burner is attached to a positioning platform with accurate and repeatable positioning both vertically and horizontally.

Horizontal scans of line-integrated spectra are collected over a spectral range from 500 to $945 \mathrm{~nm}$. This wide spectralrange necessitates two scans of the flame with different spectral grating positions. The two scan ranges are distinguishedas lower range(LR) and upper range (UR), covering from 500 to $748 \mathrm{~nm}$ and from 685 to $945 \mathrm{~nm}$, respectively.Each data acquisition consisted of a spectrum averaged from five 1-s exposures. The spectral images are summed vertically over the 0.5 -mm slit height to improve the signal-to-noiseratio. The spectra are also binned horizontally (spectrally) into $25-\mathrm{nm}$ bins. The spectrometer is calibrated with a tungsten strip filament lamp, placed coincident with the burner center and of known brightness temperature. The filament lamp is calibrated against a secondary standard photoelectric pyrometer at a wavelength of $649 \mathrm{~nm}$. The uncertainty in the spectral radiance temperature is $\pm 5 \mathrm{~K}$. The variation in emissivity of tungsten with wavelength is taken from Pon and Hessler. ${ }^{20}$ This calibration allows the spectra to be scaled to units of absolute spectral intensity (watts per cubic meter steradian).

The one-dimensional tomography is performed on the data using a three-point Abel inversion method (see Ref. 21). For each binned wavelength range, there existed an intensity scan providing experimental values of $I_{\lambda}$ vs $y$. The inversion of these data for each wavelength bin returns the local property value $G_{\lambda, r}$, which is used in Eq. (5) to calculate the radial temperature profile. When Eq. (3) is used, the absorption coefficient is obtained. The soot volume fraction is calculated using the absorption coefficient and Eq. (6).

\section{Optical Considerations}

In the development of the equations for tomographic inversion, it is implicitly assumed that the property being measured is essentially constant over the cross-sectional area sampled and that all regions along the optical axis are sampled equally. For practical line-ofsight measurements, the sampling cross section changes with position along the measurement chord. In addition, flame emission is attenuated through self-absorption while passing through the flame. Strictly speaking, this means that emission measurements are not line integrals of a local property field as is required for tomographic reconstruction. These two concerns are addressed in the following analysis.

The object focal plane is the only location in the flame where radiation emission from soot will be focused onto the entrance slit of the spectrometer. For locations on the optical axis offset from the object focal plane, the image will be located ahead or behind the slit location. As such, it is possible that the sampling efficiency of the optical system varies with position along the optical axis. It has been suggested, however, that provided the extended medium being imaged is sufficiently large in cross section to fill the field of view of the instrument, all cross sections along the optical axis are sampled equally. ${ }^{22}$ To test the optical efficiency of the present system, a strip filament lamp has been translated along the optical axis, and the resultant spectrometer signal was measured. The filament is sufficiently wide $(3 \mathrm{~mm})$ that its image overfills the entrance slit of the spectrometer. Over a 10-mm range, centered on the focal plane, the intensity, measured at all wavelengths, varies by less than $2 \%$ for f/ $16-f / 64$ optical arrangements. From this, it is concluded that the sampling efficiency is uniform for this combination of f-numbers and depths of field.

Image blurring will occur because of the extended depth of field sampled through the flame. The cross-sectional area of the sample chord at a given position in the measurement field can be quantified by noting that it is defined by the image of the entrance slit of the detector at that same location. The slit image characteristics are determined by the collection lens and by the aperture of this lens. Specifically, the image size depends on magnification, lens aberration, and aperture diffraction effects. A simple geometricoptical analysis of the depth of field ${ }^{23}$ suggests that the diameter of the sampled region increases as $x d / l=x / \&$ with displacement $x$ from the focal plane, where $l$ is the lens to source and lens to slit distances (1:1 imaging) and $d$ is the diameter of the lens aperture. For the small lens aperture (high f-numbers) used, diffraction effects from the circular aperture are more significant than lens aberrations. This increases the area of the focal plane that is viewed. Diffraction from the aperture produces an Airy pattern with a central maximum intensity disk of diameter $w_{\text {diff }}=2.44 \lambda \rho$. Lens chromaticity makes the object plane of the burner a function of wavelength, which effectively increases the required depth of field through the flame. Although this mixture of geometric and physical optical quantities is not additive, this analysis suggests an equation of the form

$$
w_{\text {theo }}(x, \lambda)=w_{0}\left\{1+w_{1}[x-f(\lambda)]^{2}\right\}^{0.5}
$$

to describe the dependence of the cross section sampled on displacement from the focus and the sampling wavelength. Here $w_{0}$ and $w_{1}$ are arbitrary constants and $f(\lambda)$ describes the shift of focus with wavelength and is specific to the lens used. This equation will be shown to provide a good empirical description of the data.

To measure the sampling cross section as a function of position on the optical axis, the spectrometer entrance slit is covered on the backplane with a sheet of light diffusion paper and backlit using a small lamp to provide a secondary source of radiation. A Cohu 4800 CCD camera located on the optical axis is translated to different offsets from the object focal plane of the burner. For each offset, an image of the entrance slit is recorded, and an apparent slit width is calculated. Figure 3 shows the relation between image width (calculated using a peak intensity equivalent width) and position on the optical axis for different $\mathrm{f}$-numbers. The peak intensity equivalent width was the spatial integral of the image intensity divided by the peak observed intensity, that is, the width of a rectangle having the same area and peak intensity as the image plot. For the depth of field of $\pm 4 \mathrm{~mm}$, it is apparent that the results for both $f / 42$ and $f / 67$ show only a small increase in image size with displacement from the focal plane. As expected, the $f / 13$ optical arrangement shows a much larger increase, but, contrary to the predictions of the simple model, there is no decrease in focal spot size. This undoubtedly results from the neglect of spherical aberration and chromaticity, which become much more important at lower f-numbers. The data are empirically fitted to Eq. (7), with the results shown as curves in Fig 3. Chromatic aberration is not considered in the empirical fit, that is, $f(\lambda) \simeq 0$, because of limitations in the lens width characterizationapparatus and because an achromatic lens was used. Even if a single-element lens 


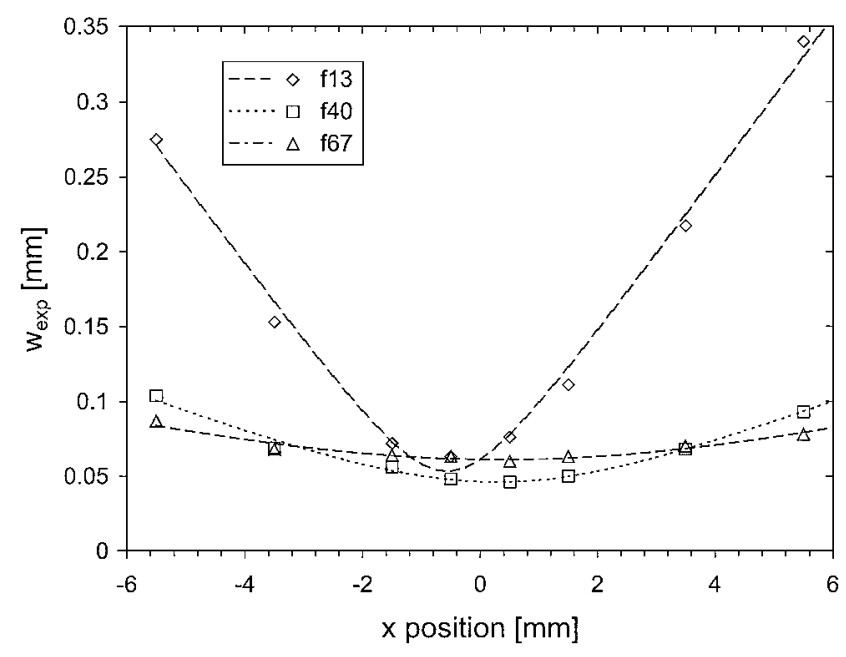

Fig. 3 Slit image width (peak equivalent width) and curve fitted to data points of form $w=w_{0}\left[1+w_{1}\left(x-w_{2}\right)^{2}\right]^{0.5}$ for three f-numbers.

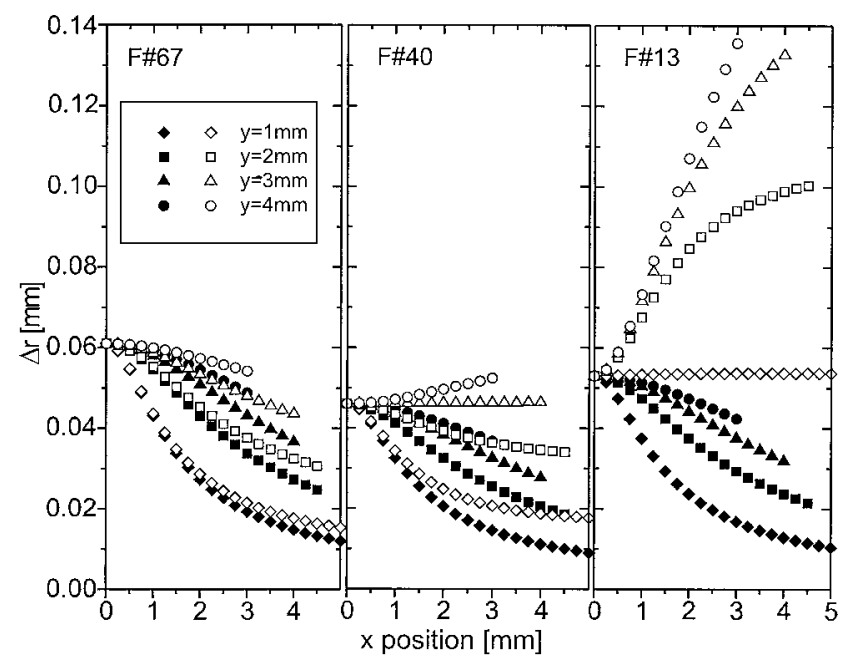

Fig. 4 Comparison of $\Delta r$ across the optical sampling width $w_{\text {exp }}$, for the optimal case of $w=w_{0}$ (solid shapes) and the experimentally determined width $w=w_{0}\left(1+w_{1} x^{2}\right)^{0.5}$ (open shapes) for three f-numbers.

had been employed, the displacement of the focal position over the wavelength range used would typically be $1 \mathrm{~mm}$, which is approximately an order of magnitude less than the required depth of field. Chromaticity is only an issue when using low f-number optics. It is observed that changes in sampling cross section are qualitatively similar to predictions from our simple analysis.

The observed variation in sampling cross section will only lead to errors in the Abel inversion if the flame properties change appreciably over the sampled cross sections. With an axisymmetric property field, it is, therefore, valuable to quantify how radial position $r$ varies across the sample cross section for a given $(x, y)$ position. Because $\Delta r / \Delta y \approx y / r$, then $\Delta r=w_{\exp } \cdot y / r$, where $\Delta r$ is the spread of radial locations sampled for a given $(x, y)$ position in the flame as a result of the finite resolution of the imaging system. In Fig. 4, the $\Delta r$ values, calculated from the empirically fit experimental equivalent width curves, are compared for different f-numbers. For $f / 67, \Delta r$ is largest at the focal plane for all $y$ positions and decreases with increased $x$. For $f / 40$, the $\Delta r$ increases with increasing $x$ for values of $y$ greater than $3 \mathrm{~mm}$. The largest $\Delta r$ value for $f / 40$ is, however, smaller than the largest $\Delta r$ value for $f / 67$. For $f / 13, \Delta r$ increases with $x$ for all values of $y$. For all f-numbers, the largest $\Delta r$ values correspond to the edges of the flame, where the highest spatial resolution is required.

The ultimate test of the optical system is in the determination of soot temperatures and absorption coefficients in the burner flame; therefore, these measurements were performed for $f / 42$ and $f / 9$. Both sets of data were collected on the same day for the same burner

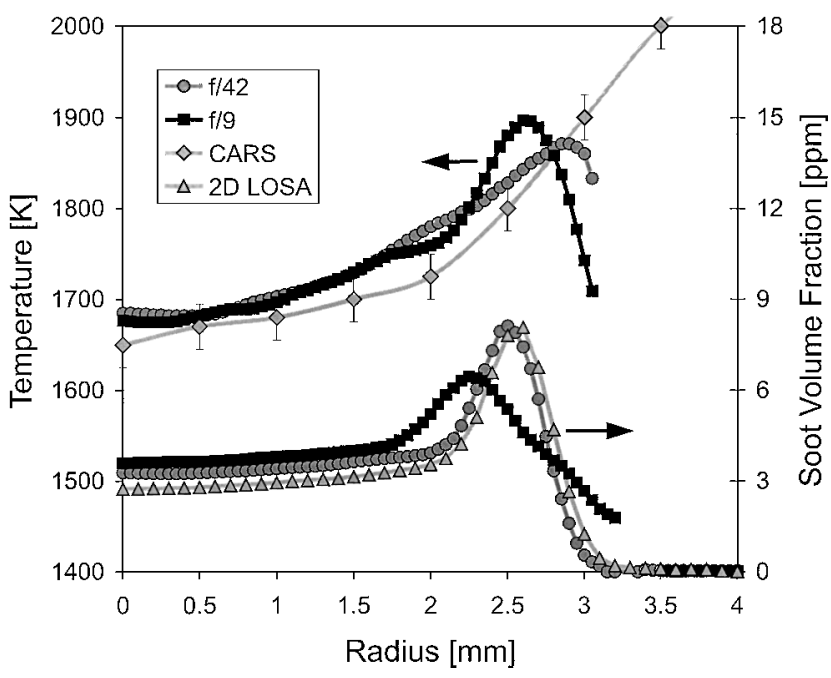

Fig. 5 Soot temperature and soot volume fraction profiles as a function of radial location in the flame at a downstream location of $30 \mathrm{~mm}$ with f/9 and f/42 optical configuration compared with CARS temperatures ${ }^{17}$ and two-dimensional LOSA scatter-corrected soot volume fractions. ${ }^{24}$

conditions. A measurement height of $30 \mathrm{~mm}$ above the burner tip was chosen because, at this position, the flame diameter is moderate (approximately $6 \mathrm{~mm}$ ), and the reaction zone is relatively narrow with large soot concentration gradients.

The results are summarized in Fig. 5 with comparisons to CARS temperature measurements and soot volume fraction measured by two-dimensional line-of-sight light attenuation (LOSA) corrected for scatter. The CARS ${ }^{17}$ and two-dimensional LOSA ${ }^{24}$ techniques have been described previously. The CARS temperature and $f / 42$ soot temperature measurements are consistent in trend out to the edge of the flame, where soot concentrations and, therefore, emission signals drop rapidly. Both the temperature and soot volume fraction results for $f / 9$ are in marked disagreement to the $f / 42$ data and the CARS/two-dimensionalLOSA data. The temperature curve for $f / 9$ showed deviations beginning at $r=1.7 \mathrm{~mm}$, where there is a dip followed by rise in temperature, which is not physically justifiable. Both the peak temperature and the peak soot concentration are shifted toward the flame center, and the latter is $20 \%$ lower than the two-dimensional LOSA values. The differences noted in the $\mathrm{f} / 9$ results are particularly marked for $r=1.7-3.0 \mathrm{~mm}$, where the spread in $\Delta r$ values (Fig. 4) and the temperature and soot concentration gradients are largest. These errors undoubtedly result from the decrease in spatial resolution coupled with a possible chromatic shift of focus. The former allows larger variation in $\Delta r$ across the sampling cross section for sampling chords near the edge of the flame (Fig. 4); the latter allows for different sampling paths along the measurement chord as a function of wavelength. This results in temperature and soot volume fraction profiles that are incorrect and not merely broadened versions of the true profile.

The effects of lens chromaticity and image blur were also modeled by numerically simulating the line-integrated radiation measurements and then analyzing the modeled emission using the same technique used for measured data, which has been described. The radiation contribution from a particular $x$ location $x_{0}$ for a chord at position $y_{0}$ was approximated as the average of the values at $y_{0}+w_{\text {theo }}\left(x_{0}, \lambda\right) / 2$ and $y_{0}-w_{\text {theo }}\left(x_{0}, \lambda\right) / 2$, where $w\left(x_{0}, \lambda\right)$ is defined in Eq. (7). Chromatic effects were simulated by a linear variation of $f(\lambda)$ with $\lambda$. The results of this simulation show that at higher f-numbers, $f / 40-\wp / 67$, the calculated soot temperatures and concentrationsare largely insensitive to chromatic aberrations or focal position changes. However, at lower f-numbers, where the depth of field is much smaller, marked errors can arise. This is particularly so if there is chromatic aberration or if the focal plane is not located in the center of the flame. With a reasonable combination of chromaticity and focal displacement, results similar to those for f/9 in Fig. 5 are obtained.

There is very good agreement in soot concentration data from the two-dimensional light attenuation and $\mathrm{f} / 42$ soot emission 
temperature methods. Based on this observation, it is concluded that operation at $\mathrm{f} / 42$ or higher is necessary for accurate and high spatial resolution measurement of soot temperature and soot volume fraction for the thickness of flame and slit width chosen for this experiment.

In addition to sampling problems, there is also error introduced into the radiation emission measurements due to emission attenuation. Soot is both a good emitter and absorber of radiation, and so a portion of the light emission radiating along the measurement chord is reabsorbed by soot particles. The percentage of emission attenuation is dependent on the position of the emitter along the chord through the flame. This creates several problems. First, the attenuation of emission will necessarily introduce errors in the line-of-sight spectral emission measurement. Second, because each emitter does not experience the same percentage attenuation, the line-of-sight measurements become technically unsuitable for tomographic inversion using the Abel inversion. Finally, the emission attenuation is also wavelength dependent, which will shift the slopes of the spectral intensity measurements and, hence, introduce error into the temperatures derived from them.

To determine the effect of these sources of error in the present measurements, the radiation emission along a chord of a flame as

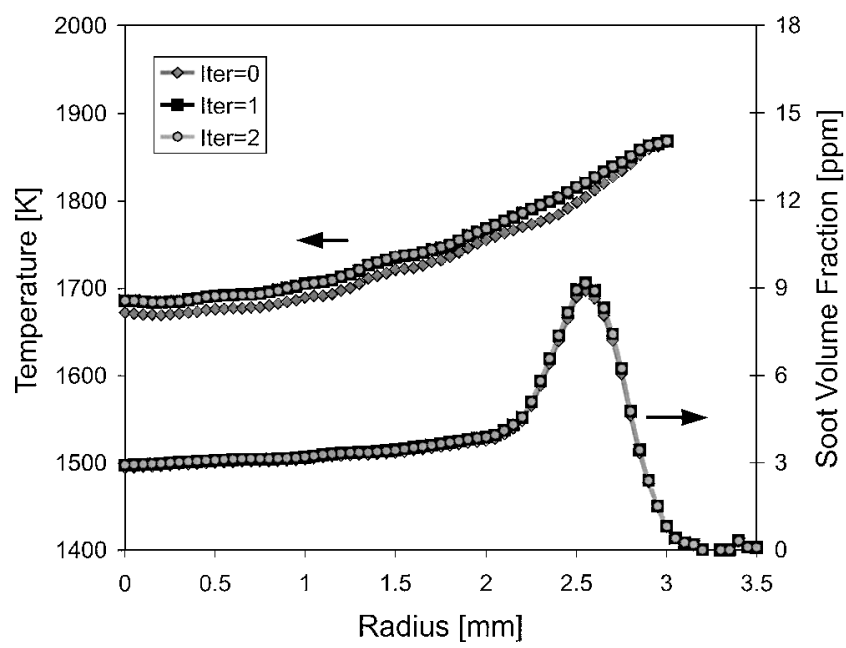

Fig. 6 Comparison of the soot surface temperatures obtained from emission spectroscopy with (iteration 1 and iteration 2) and without (iteration 0) attenuation correction in the flame at a downstream location of $30 \mathrm{~mm}$ and soot volume fraction data with and without attenuation correction. represented by Eq. (1) is modeled including and not including the extinction term present. It is then possible to correct the measured emission through the relationship

$$
I_{\text {corr }}(y, \lambda)=\frac{I_{\text {meas }} \cdot I_{\text {mod }_{\text {noext }}}}{I_{\text {modext }_{\text {ext }}}}
$$

where the modeled emission is calculated for any radial offset and wavelength value for which measurements are taken. The modeled intensity is calculated using inferred temperature and absorption coefficients obtained from an analysis of the measurements, ignoring attenuation effects. Extinction coefficients are estimated from absorption coefficients through the relationship $K_{\lambda}^{(e)}=N_{P} \alpha_{\lambda}^{(a)}\left(1+\rho_{\mathrm{sa}}\right)$, where $\rho_{\mathrm{sa}}$ is estimated using RDG/PFA theory as described in the Results and Discussion section.

In Fig. 6, the temperature and soot volume fraction for a height of $30 \mathrm{~mm}$ are shown with attenuation correction applied in the first and second iteration. The solution converged with a single iteration. Note that the influence of the attenuationerror is very small; however, the correction technique holds promise for application to flames with higher soot loading.

\section{Results and Discussion}

Scans of the flame were performed at heights of 10,20,30, and $50 \mathrm{~mm}$ above the fuel outlet. In Fig. 7, temperature results [calculated assuming a constant $E(m)$ with wavelength] are summarized and compared to CARS nitrogen gas temperature measurements. Soot temperature results are only included for regions where the soot concentration is sufficiently high to give reliable radiation signal levels. Error bars on CARS data indicate the precision of those measurements $\pm 25-50 \mathrm{~K}$, with the upper limit applicable to regions of high soot concentration. Within these limits, most of the soot temperatures are in agreement with CARS temperatures.

Also shown in Fig. 7 is the soot volume fraction $f_{v}$ calculated using Eq. (6) and an $E(m)$ of 0.26 . The results are compared to two-dimensionalLOSA measurements obtained on the same burner, which were first converted to absorption coefficients by correcting for scattering ${ }^{24}$ The experimental error in the two-dimensional LOSA measurements [neglecting the uncertainty in the absolute value of $E(m)]$ is a combination of uncertainties in the scattering correction and experimental uncertainties in the transmission measurements. As noted in the Theory section, the value of $E(m)$ is arbitrary, and for a comparison of the two measurement techniques of soot concentration to be valid, the same value of $E(m)$ must be used. The present results offer no information as its absolute value. The experimental uncertainties in soot concentration resulting from uncertainty in the transmission measurements are a function of radial position. ${ }^{21}$ The errors were estimated ${ }^{21,24}$ to be

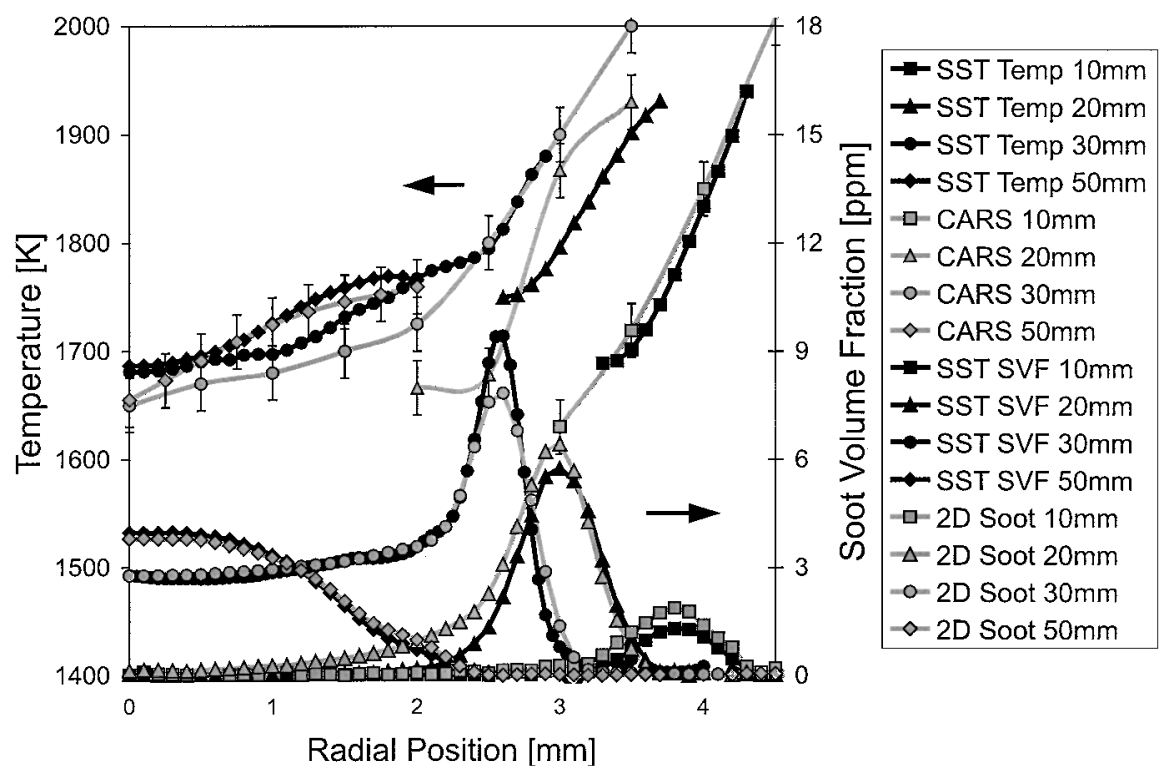

Fig. 7 Soot temperature and soot volume fraction profiles as a function of radial location in the flame at downstream locations of $10,20,30$, and $50 \mathrm{~mm}$ compared with CARS temperatures ${ }^{17}$ and two-dimensional LOSA scatter-corrected soot volume fractions. ${ }^{24}$ 
$\pm 0.04 \mathrm{ppm}$ for $r>2.0 \mathrm{~mm}$ and $\pm 0.08 \mathrm{ppm}$ for $r=0.5 \mathrm{~mm}$. The scattering correction is based on the primary particle and agglomerates size measured in a burner of identical construction to ours and with a slightly higher fuel flow rate. ${ }^{25}$ The flame height for this higher flow rate was $88 \mathrm{~mm}$, somewhat greater than the $67 \mathrm{~mm}$ we observed. The data of Dobbins and Megaridis ${ }^{25}$ are scaled by the ratio of these flame heights in applying it to our flames. The scattering correction term $\rho_{\mathrm{sa}}$ is calculated from RDG/PFA theory following the formulation of Köylü ${ }^{8}$ and using a fractal prefactor $k_{f}=8.5$, a fractal dimension $D_{f}=1.74$, and a geometric mean standard deviation for the assumed lognormal distribution of 2.4. The correction results in a reduction of the soot volume fraction of $2,12,16$, and $9 \%$ for heights of $10,20,30$, and $50 \mathrm{~mm}$, respectively. Although this correction procedure carries some uncertainties, the corrections are quite small. With these two sources of error, the error in the two-dimensional LOSA-based soot concentrations is estimated to be no more than $\pm 10 \%$.

Lower in the flame, the two-dimensional measurements indicate consistently higher soot volume fraction particularly toward the burner centerline. It is speculated that there may be light attenuation in these regions, which did not originate from soot particles, which biases the calculated soot volume fraction. We have unpublished results comparing laser-induced incandescence (LII) with two-dimensional LOSA that supports this interpretation.

At $30 \mathrm{~mm}$ above the burner outlet, the agreement is very good in the core of the flame, with a $15 \%$ higher peak in the annulus for the soot volume fractions inferred from soot emission measurements. This difference is possibly due to higher spatial resolution in the current technique, or an underestimate of the soot temperature in the annulus region at the $30-\mathrm{mm}$ height. At $50 \mathrm{~mm}$, the agreement between the two techniques is excellent. Agreement of the soot concentrations obtained from soot emission temperature measurements and two-dimensionalLOSA corrected for scattering is further support for an $E(\mathrm{~m})$ independent of wavelength from 500 to $945 \mathrm{~nm}$.

A comparison of the present results with those obtained by CARS (temperature measurements) and two-dimensionalLOSA (soot volume fraction) can be used to set limits on the spectral dependence of $E(m)$. To investigate the limits over which there is a satisfactory agreement among the various data sets, we assume a functional form for the variation of $E(m)$ with wavelength of the form

$$
E(m)=0.26+C(\lambda-577) / 500
$$

The two-dimensional LOSA measurements were performed at $577 \mathrm{~nm}$, and the form of Eq. (9) is such that $E(m)$ at $577 \mathrm{~nm}$ remains constant at 0.26 . The temperature and soot concentration comparison for the data at heights of 30 and $50 \mathrm{~mm}$ are shown in Figs. 8 and 9 , respectively, for $C$ values of $-0.052,0$, and 0.052 . These $C$ values correspond to a range of $E(m)$ variations over the wavelength we have used (500-945 nm) of approximately plus or minus $20 \%$. Note that the emission measurements were performed for two spectral ranges LR (500-748 $\mathrm{nm})$ and UR (685-945 nm).

From Figs. 8 and 9, we see that a $C$ value of 0 gives the best agreement among the various data sets with the possible exception of the soot volume fraction measurements in Fig. 9, where a $C$ value between 0 and 0.052 would provide the best fit. The agreement among the data sets is within the combined error limits for $C=0.052$. However, for $C=-0.052$ the data sets are in clear disagreement.

Temperature calculations were also made using $E(m)$ based on the dispersion relationshipof Dalzell and Sarofim. ${ }^{16}$ This gives temperatures almost identical to those in Fig. 5 for the lower wavelength range, but approximately $50 \mathrm{~K}$ higher for the upper wavelength range. These higher temperatures are in clear disagreement with the CARS data.

For the wavelength range $500-650 \mathrm{~nm}$, the $C$ value of 0 is supported by earlier work. ${ }^{16,26-28}$ The present results extend this conclusion to the wavelength range 500-945-nm, and the error limits imply that $E(m)$ increases by no more than $20 \%$ over this wavelength range. Thus, dispersed soot radiation in the wavelength range 500-945 $\mathrm{nm}$ can be used to determine soot temperature with good reliability. This result is important for characterizing flame temperatures and for the absolute intensity self-calibration of LII. ${ }^{29}$
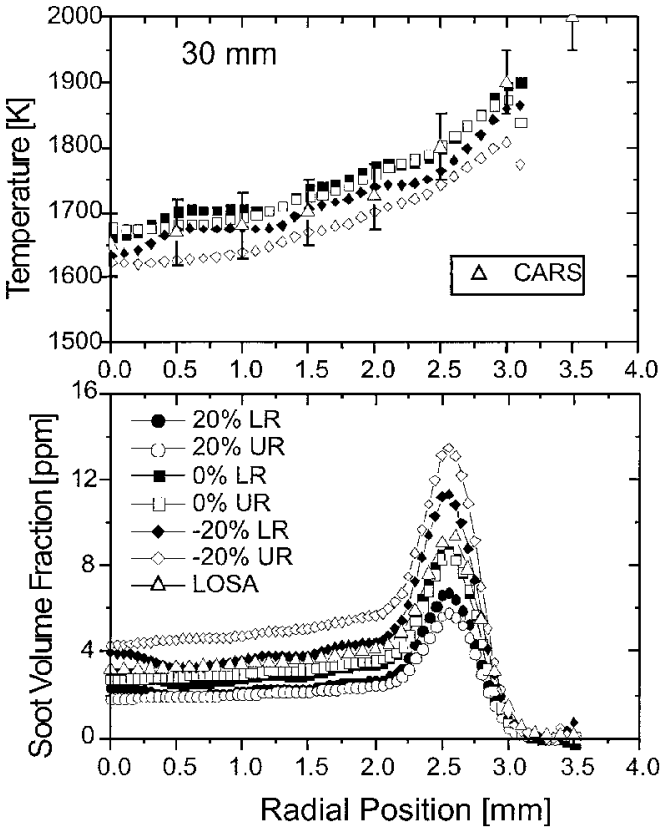

Fig. 8 Effect of refractive index absorption function $E(m)$ on soot temperature and soot volume fraction evaluated from measurements at a flame downstream location of $30 \mathrm{~mm}: x \% \mathrm{LR}$ (UR) refers to values evaluated by applying a $x \%$ change of $E(m)$ using the measurements taken by the LR (UR) scan; open triangles denote CARS temperature measurements ${ }^{17}$ (top) and LOSA soot volume fraction measurements ${ }^{24}$ (bottom).
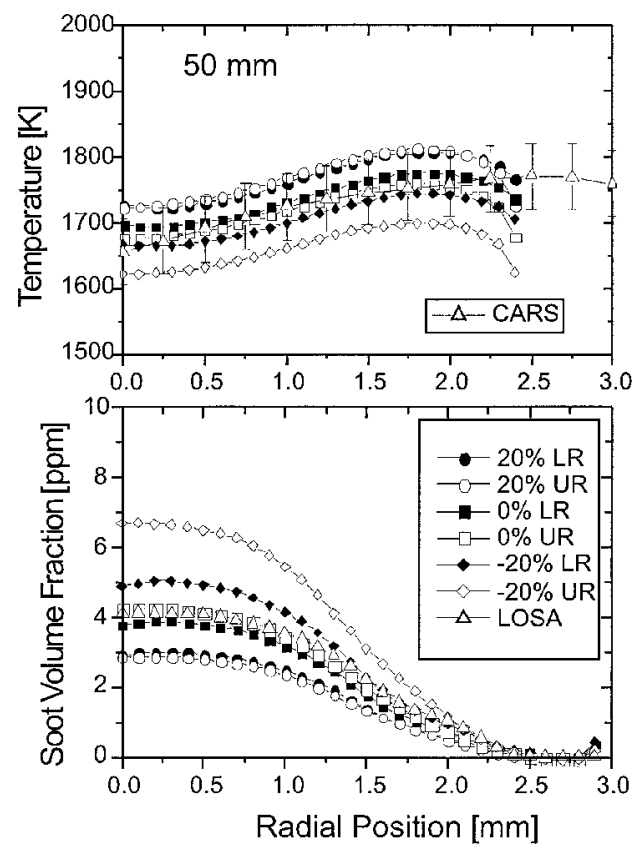

Fig. 9 Effect of refractive index absorption function $E(m)$ on soot temperature and soot volume fraction evaluated from measurements at flame downstream location of $50 \mathrm{~mm}: x \%$ LR (UR) refers to values evaluated by applying a $x \%$ change to $E(m)$ using the measurements taken by the LR (UR) scan; open triangle symbols denote CARS temperature measurements ${ }^{17}$ (top) and LOSA soot volume fraction measurements ${ }^{24}$ (bottom).

\section{Conclusions}

A technique has been developed for high-resolution determination of soot temperature and soot volume fraction. The optical constraints necessary for correct sampling of the flame have been considered. The important parameters are depth of field, slit width, and f-number. Errors in cross-sectional sampling area have the largest influence at the edges of the flame, where the highest resolution is required. Because of the implicit requirements of the Abel inversion, errors in sampling area can produce errors in measurements, 
rather than simply creating a spatial average. Experimental observations support these conclusions and indicate that an $f / 40$ or higher light collection system is required for accurate measurements in the present laminar diffusion flame when using a $25-\mu \mathrm{m}$ spectrometer slit. For the soot loading and temperaturesobserved in this flame, the influence of emission attenuation is small; however, the correction technique presented has good potentialfor measurement corrections in more heavily sooting flames. Soot temperatures are in agreement with CARS temperature measurements. This agreementimplies that the assumption of constant $E(m)$ over the wavelength range 500$945 \mathrm{~nm}$ is substantially correct. The assumption is further justified by the good agreement between soot volume fractions as measured by soot emission temperature and two-dimensionalLOSA at heights above $20 \mathrm{~mm}$. By investigating the effect of an $E(m)$ that varies linearly with wavelength, we conclude that $E(m)$ does not increase by more than $20 \%$ over the 500-945-nm range studied. Furthermore, a $20 \%$ decrease in $E(m)$ over this wavelength range is clearly inconsistent with the data. This refined soot temperature measurement technique offers a means for high-resolution measurement of temperature and soot volume fraction in the laminar diffusion flame, which will be invaluable for the generation of flamelet libraries and for understanding of soot formation mechanisms in flames.

\section{References}

${ }^{1}$ Hall, R. J., and Bonczyk, P. A., "Sooting Flame Thermometry Using Emission/Absorption Tomography," Applied Optics, Vol. 29, No. 31, 1990, pp. 4590-4598.

${ }^{2}$ Choi, M. Y., Hamins, A., Mulholland, G. W., and Kashiwagi, T., "Simultaneous Optical Measurement of Soot Volume Fraction and Temperature in Premixed Flames," Combustion and Flame, Vol. 99, No. 1, 1994, pp. 174186.

${ }^{3}$ Zhao, H., and Ladommatos, N., "Optical Diagnostics for Soot and Temperature Measurement in Diesel Engines," Progress in Energy and Combustion Science, Vol. 24, No. 3, 1998, pp. 221-255.

${ }^{4}$ Klassen, M., and Gore, J. P., "Temperature and Soot Volume Fraction Statistics in Toluene-Fired Pool Fires," Combustion and Flame, Vol. 93, No. 3, 1993, pp. 270-278.

${ }^{5}$ Klassen, M., Gore, J. P., Sivathanu, Y. R., Hamins, A., and Kashiwagi, "Radiative Heat Feedback in a Toluene Pool Fire," Twenty-Fourth Symposium (International) on Combustion, Combustion Inst., Pittsburgh,PA, 1992, pp. 1713-19.

${ }^{6}$ DeIuliis, S., Barbini, M., Benecchi, S., Cignoli, F., and Zizak, G., "Determination of the Soot Volume Fraction in an Ethylene Diffusion Flame by Multiwavelength Analysis of Soot Radiation," Combustion and Flame, Vol. 115, No. 1-2, 1998, pp. 253-261.

${ }^{7}$ Porter, R. W., "Numerical Solution for Local Emission Coefficients in Axisymmetric Self-Absorbed Sources," SIAM Review, Vol. 6, No. 6, 1964, pp. 228-242.

${ }^{8}$ Köylü, Ü. Ö., "Quantitative Analysis of In Situ Optical Diagnostics for Inferring Particle/Aggregate Parameters in Flames: Implications for Soot Surface Growth and Total Emissivity," Combustion and Flame, Vol. 109, No. 3, 1997, pp. 488-500.

${ }^{9}$ Köylü, Ü. Ö., and Faeth, G. M., "Optical Properties of Overfire Soot in Buoyant Turbulent Diffusion Flames at Long Residence Times," Journal of Heat Transfer, Vol. 116, No. 1, 1994, pp. 152-159.

${ }^{10}$ Köylü, Ü. Ö., and Faeth, G. M., "Optical Properties of Soot in Buoyant Laminar Diffusion Flames,” Journal of Heat Transfer, Vol. 116, No. 4, 1994 pp. 971-979.

${ }^{11}$ Bonczyk, P. A., and Hall, R. J., "Fractal Properties of Soot Agglomer- ates," Langmuir, Vol. 7, No. 6, 1991, pp. 1274-1280.

${ }^{12}$ Dobbins, R. A., and Megaridis, C. M., "Absorption and Scattering of Light by Polydisperse Aggregates," Applied Optics, Vol. 30, No. 33, 1991, pp. 4747-4754.

${ }^{13}$ Dobbins, R. A., Santoro, R. J., and Semerjian, H. G., "Analysis of Light Scattering from Soot Using Optical Cross Sections for Aggregates," Proceedings of the Combustion Institute, Vol. 23, Combustion Inst., Pittsburgh, PA, 1990, pp. 1525-1532.

${ }^{14}$ Smyth, K. C., and Shaddix, C. R., "Elusive History of $m=1.57-0.56 i$ for the Refractive Index of Soot," Combustion and Flame, Vol. 107, No. 3 1996, pp. 314-320.

${ }^{15} \mathrm{Wu}$, J. S., Krishnan, S. K., and Faeth, G. M., "Refractive Indices at Visible Wavelengths of Soot Emitted from Buoyant Turbulent Diffusion Flames," Proceedings of the 199631 st ASME National Heat Transfer Conference, Vol. 325, American Society of Mechanical Engineers, Fairfield, NJ, 1996 , pp. 83-93.

${ }^{16}$ Dalzell, W. H., and Sarofim, A. F., "Optical Constants of Soot and Their Application to Heat Flux Calculations," Journal of Heat Transfer, Vol. 91 Feb. 1969, pp. 100-104.

${ }^{17}$ Gülder, Ö. L., Snelling, D. R., and Sawchuk, R. A., "Influence of Hydrogen Addition to Fuel on Temperature Field and Soot Formation in Diffusion Flames," Proceeding sof the Combustion Institute, Vol. 26, Combustion Inst. Pittsburgh, PA, 1996, pp. 2351-2357.

${ }^{18}$ Santoro, R. J., Yeh, T. T., Horvath, J. J., and Semerjian, H. G., "The Transport and Growth of Soot Particles in Laminar Diffusion Flames," Combustion Science and Technology, Vol. 53, No. 2-3, 1987, pp. 89-115.

${ }^{19}$ Mewes, B. S., and Seitzman, J. M., "Soot Volume Fraction and Particle Size Measurements with Laser-Induced Incandescence," Applied Optics, Vol. 36, No. 3, 1997, pp. 709-717.

${ }^{20}$ Pon, R. M., and Hessler, J. P., "Spectral Emissivity of Tungsten: Analytic Expressions for the 340-nm to 2.6-Micron Spectral Region," Applied Optics Vol. 23, No. 7, 1984, pp. 975-976.

${ }^{21}$ Dasch, C. J., "One-Dimensional Tomography: A Comparison of Abel, Onion-Peeling, and Filtered Backprojection Methods," Applied Optics, Vol. 31, No. 8, 1992, pp. 1146-1152.

${ }^{22}$ Sawyer, R. A., Experimental Spectroscopy, 3rd ed., Dover, New York, 1963, pp. 37-49

${ }^{23}$ Smith, W. J., Modern Optical Engineering, 2nd ed., McGraw-Hill, New York, 1990,pp. 145-150.

${ }^{24}$ Snelling, D. R., Thomson, K. A., Smallwood, G. J., and Gülder, Ö. L., "Two-Dimensional Imaging of Soot Volume Fraction in Laminar Diffusion Flames," Applied Optics, Vol. 38, No. 12, 1999, pp. 2478-2485.

${ }^{25}$ Dobbins, R. A., and Megaridis, C. M., "Morphology of FlameGenerated Soot as Determined by Thermophoretic Sampling," Langmuir Vol. 3, No. 2, 1987, pp. 254-259.

${ }^{26}$ Köylü, Ü. Ö., and Faeth, G. M., "Spectral Extinction Coefficients of Soot Aggregates from Turbulent Diffusion Flames," Journal of Heat Transfer, Vol. 118, No. 2, 1996, pp. 415-421.

${ }^{27}$ Krishnan, S. S., Lin, K. C., and Faeth, G. M., "Optical Properties in the Visible of Overfire Soot in Large Buoyant Turbulent Diffusion Flames," Journal of Heat Transfer, Vol. 122, No. 3, 2000, pp. 517-524.

${ }^{28}$ Stagg, B. J., and Charalampopoulos, T. T., "Refractive Indices of Pyrolytic Graphite, Amorphous Carbon, and Flame Soot in the Temperature Range 25 Degree to 600 Degree C," Combustion and Flame, Vol. 94, No. 4 1993, pp. 381-396.

${ }^{29}$ Snelling, D. R., Smallwood, G. J., Gülder, Ö. L., Liu, F., and Bachalo, W. D., "A Calibration-Independent Technique of Measuring Soot by Laser Induced Incandescence Using Absolute Light Intensity," Proceedings of the Second Joint Meeting of the US Sections of the Combustion Institute, Oakland, CA, 2001. 